\title{
MHD Transient Flow with Hall Current Past an Accelerated Horizontal Porous Plate in a Rotating System
}

\author{
Nazibuddin Ahmed ${ }^{1}$, Jiwan Krishna Goswami ${ }^{2}$, Dhruba Prasad Barua ${ }^{1}$ \\ ${ }^{1}$ Department of Mathematics, Gauhati University, Guwahati, India \\ ${ }^{2}$ Department of Mathematics, ADP College, Nagaon, India \\ Email: saheel_nazib@yahoo.com,jkg_gurs@rediffmail.com,math_byte@yahoo.com
}

Received September 3, 2013; revised October 3, 2013; accepted October 10, 2013

Copyright (C) 2013 Nazibuddin Ahmed et al. This is an open access article distributed under the Creative Commons Attribution License, which permits unrestricted use, distribution, and reproduction in any medium, provided the original work is properly cited.

\begin{abstract}
An exact solution to the problem of an MHD transient flow with Hall current past a uniformly accelerated horizontal porous plate in a rotating system has been presented. The dimensionless governing equations of the flow problem are solved by Laplace transform technique in closed form. A uniform magnetic field is assumed to be applied transversely to the direction of the flow. The expressions for velocity fields and skin-frictions are obtained in non-dimensional form. The primary and secondary velocity distributions and skin-frictions at the plate due to primary and secondary velocity field are demonstrated graphically and the effects of the different parameters namely, rotational parameter, Hartmann number, Hall parameter and acceleration parameter are discussed and the results are physically interpreted.
\end{abstract}

Keywords: MHD; Rotating System; Transient; Hall Current; Mass Transfer; Skin-Friction

\section{Introduction}

Many engineering problems are susceptible to MHD analysis. The study of MHD flow problems has achieved remarkable interest due to its application in MHD generators, MHD pumps and MHD flow meters etc. The study of effects of magnetic field on free convection flow is important in liquid metals, electrolytes and ionized gases. Geophysics encounters MHD phenomena in interactions of conducting fluids and magnetic fields. The rotating flow of an electrically conducting fluid in presence of magnetic field has got its importance in Geophysical problems. The study of rotating flow problems are also important in the solar physics dealing with the sunspot development, the solar cycle and the structure of rotating magnetic stars. It is well known that a number of astronomical bodies possess fluid interiors and magnetic fields. Changes that take place in the rate of rotation, suggest the possible importance of hydro magnetic spinup. The general theory of rotating fluids has received growing interest during last decade because of its application in Cosmic and Geophysical science. In this regard, we may cite the works done by Raptis [1], Singh [2,3], Alam et al. [4] and Debnath [5].
MHD in the present form is due to the pioneer contri bution of several notable authors like Alfven [6], Cowling [7], Ferraro and Pulmpton [8] etc. It was emphasized by Cowling (1975) that when the strength of the applied magnetic field is sufficiently large, Ohm's law needs to be modified to include Hall current. The Hall effect is due merely to the sideways magnetic force on the drifting free charges. The electric field has to have a component transverse to the direction of the current density to balance this force. In many works of Plasma physics, it is not paid much attention to the effect caused due to Hall current. However, the Hall effect can not be completely ignored if the strength of the magnetic field is high and the number density of electrons is small as it is responsible for the change of the flow pattern of an ionized gas. Hall effect results in a development of an additional potential difference between opposite surfaces of a conductor for which a current is induced perpendicular to both the electric and magnetic field. This current is termed as Hall current. It was discovered in 1879 by Edwin Herbert Hall while working on his doctoral degree at the Johns Hopkins University in Baltimore, Maryland, USA. Pop [9], Kinyanjui et al. [10], Archrya et al. [11] 
and Ahmed and Kalita [12] etc. have presented some model studies on the effect of Hall current on MHD convection flow because of its possible application in the problems of MHD generators and Hall accelerators. An unsteady MHD free convective flow past a vertical porous plate immersed in a porous medium with Hall current, thermal diffusion and heat transfer have been studied by Ahmed et al. [13]. Recently, Ahmed and Sarmah [14] have carried out an investigation of MHD transient flow past an impulsively started infinite horizontal porous plate in a rotating system with Hall current.

Due to the importance of studying MHD flow problems in rotating fluid with Hall current, we have proposed in the present paper to investigate an unsteady MHD transient flow with Hall currents past a uniformly accelerated porous plate in a rotating system.

\section{Basic Equations}

The equations governing the motion of an incompressible viscous electrically conducting fluid in a rotating system in presence of a magnetic field are as under:

Equation of continuity:

$$
\nabla \cdot \boldsymbol{q}=0
$$

Momentum equation:

$$
\begin{aligned}
& \rho\left[\frac{\partial \boldsymbol{q}}{\partial t^{\prime}}+2 \boldsymbol{\Omega} \times \boldsymbol{q}+\boldsymbol{\Omega} \times(\boldsymbol{\Omega} \times \boldsymbol{r})+(\boldsymbol{q} \cdot \nabla) \boldsymbol{q}\right] \\
& =-\nabla p+\boldsymbol{J} \times \boldsymbol{B}+\mu \nabla^{2} \boldsymbol{q}
\end{aligned}
$$

Kirchhoff's first law:

$$
\nabla \cdot \boldsymbol{J}=0
$$

General Ohm's law:

$$
\begin{aligned}
& \boldsymbol{J}+\frac{\omega_{e} \tau_{e}}{B_{0}}(\boldsymbol{J} \times \boldsymbol{B}) \\
& =\sigma\left[\boldsymbol{E}+\boldsymbol{q} \times \boldsymbol{B}+\frac{1}{e \eta_{e}} \nabla p_{e}\right]
\end{aligned}
$$

Gauss's law of magnetism:

$$
\nabla \cdot \boldsymbol{B}=0
$$

where $\boldsymbol{q}$ is the velocity vector, $\Omega$ the angular velocity of the fluid, $r$ the position vector of the fluid particle $P$ considered, $\rho$ the fluid density, $\mathrm{p}$ the pressure, $\boldsymbol{J}$ the current density, $\boldsymbol{B}$ the magnetic induction vector, $\mu$ the co-efficient of viscosity, $\sigma$ the electrical conductivity, $t^{\prime}$ the time, $B_{0}$ the strength of the applied magnetic field, $\omega_{e}$ the electron frequency, $\tau_{e}$ the electron collision time, $e$ the electron charge, $\eta_{e}$ the number density of electron, $p_{e}$ the electron pressure, $\boldsymbol{E}$ the electric field, $2 \Omega \times \boldsymbol{q}$ is the Coriolis acceleration, $\boldsymbol{\Omega} \times(\boldsymbol{\Omega} \times \boldsymbol{r})$ is the centripetal acceleration and the other

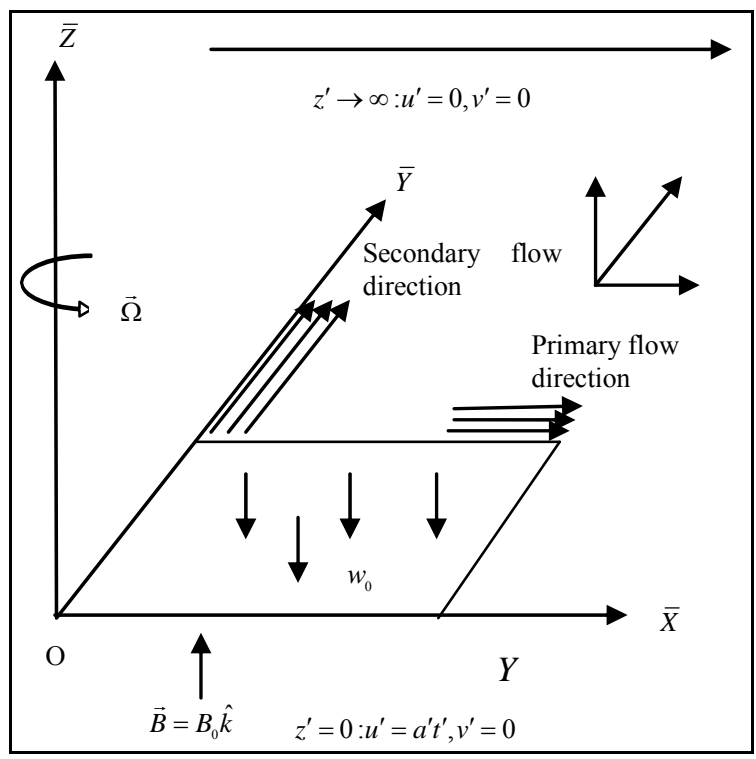

Flow configuration

symbols have their usual meanings and the other symbols have their usual meanings.

We now consider an unsteady flow of an incompressible viscous electrically conducting fluid past a suddenly started infinite horizontal porous plate relative to a rotating system with constant suction in presence of a uniform transverse magnetic field taking into account the effect of Hall current. Our investigation is restricted to the following assumptions:

- All the fluid properties are constants and the buoyancy force has no effect on the flow.

- The plate is electrically non-conducting.

- The entire system is rotating with angular velocity $\Omega$ about the normal to the plate and $|\Omega|$ is so small that $|\Omega \times(\Omega \times \boldsymbol{r})|$ can be neglected.

- The magnetic Reynolds number is so small that the induced magnetic field can be neglected.

- $p_{e}$ is constant.

- $\boldsymbol{E}=\mathbf{0}$.

Initially the plate and the fluid were rotating in unison with a constant angular velocity $\Omega$ about the normal to the plate. At time $t^{\prime}>0$, the plate is moved in its own plane relative to the rotating system with acceleration $a^{\prime}$.We introduce a coordinate system $\left(x^{\prime}, y^{\prime}, z^{\prime}\right)$ with $\mathrm{X}$-axis horizontally in the direction of the plate velocity, $\mathrm{Y}$-axis horizontally perpendicular to the direction of the plate velocity and $\mathrm{Z}$-axis along the normal to the plate which is the axis of rotation. Let $q=\hat{i} u^{\prime}+\hat{j} v^{\prime}+\hat{k} w^{\prime}$ be the fluid velocity, $\boldsymbol{J}=J_{x}^{\prime} \hat{i}+J_{y}^{\prime} \hat{j}+J_{z}^{\prime} \hat{k}$ be the current density at the point $P\left(x^{\prime}, y^{\prime}, z^{\prime}, t^{\prime}\right)$ and $\boldsymbol{B}=B_{0} \hat{k}$ be the applied magnetic field, $\hat{i}, \hat{j}, \hat{k}$ being the unit vectors along $\mathrm{X}$-axis, $\mathrm{Y}$-axis and $\mathrm{Z}$-axis respectively. As the plate is infinite in $\mathrm{X}$-direction and $\mathrm{Y}$-direction, therefore 
all the quantities except possibly the pressure are independent of $x^{\prime}$ and $y^{\prime}$.

The Equation (1) gives

$$
\frac{\partial w^{\prime}}{\partial z^{\prime}}=0
$$

which is trivially satisfied by

$$
\bar{w}=-w_{0}^{\prime}
$$

Therefore the velocity vector $\boldsymbol{q}$ is given by

$$
\boldsymbol{q}=u^{\prime} \hat{i}+v^{\prime} \hat{j}-w_{0}^{\prime} \hat{k}
$$

The Equation (5) is satisfied by

$$
\boldsymbol{B}=B_{0} \hat{k}
$$

The Equation (3) reduces to

$$
\frac{\partial J_{z}^{\prime}}{\partial z^{\prime}}=0
$$

which shows that

$$
J_{z}^{\prime}=0
$$

(as the plate is electrically non-conducting).

Hence the current density is given by

$$
\boldsymbol{J}=J_{x}^{\prime} \hat{i}+J_{y}^{\prime} \hat{k}
$$

Under the above assumptions, the Equation (4) takes the form:

$$
\boldsymbol{J}+\frac{m}{B_{0}}(\boldsymbol{J} \times \boldsymbol{B})=\sigma(\boldsymbol{q} \times \boldsymbol{B})
$$

where $m=\omega_{e} \tau_{e}$ is the Hall parameter.

The Equations (8)-(12) yield,

$$
\begin{aligned}
& J_{x}^{\prime}=\frac{\sigma B_{0}\left(v^{\prime}+m u^{\prime}\right)}{1+m^{2}} \\
& J_{y}^{\prime}=\frac{\sigma B_{0}}{1+m^{2}}\left(m v^{\prime}-u^{\prime}\right)
\end{aligned}
$$

With the foregoing assumptions and under the usual boundary layer approximation the Equation (2) reduces to

$$
\begin{aligned}
& \frac{\partial u^{\prime}}{\partial t^{\prime}}-w_{0}^{\prime} \frac{\partial u^{\prime}}{\partial z^{\prime}}-2 \Omega^{\prime} v^{\prime}=v \frac{\partial^{2} u^{\prime}}{\partial z^{\prime 2}}+\frac{\sigma B_{0}^{2}\left(m v^{\prime}-u^{\prime}\right)}{\rho\left(1+m^{2}\right)} \\
& \frac{\partial v^{\prime}}{\partial t^{\prime}}-w_{0}^{\prime} \frac{\partial v^{\prime}}{\partial z^{\prime}}+2 u^{\prime} \Omega^{\prime}=v \frac{\partial^{2} v^{\prime}}{\partial z^{\prime 2}}-\frac{\sigma B_{0}^{2}\left(m u^{\prime}+v^{\prime}\right)}{\rho\left(1+m^{2}\right)}
\end{aligned}
$$

with $\frac{\partial p^{\prime}}{\partial z^{\prime}}=0$.

where $w_{0}^{\prime}$ is the constant suction velocity and $v$ is the kinematic viscosity

The relevant initial and boundary conditions are

$$
\left.\begin{array}{l}
u^{\prime}=0, v^{\prime}=0 \text { for } t^{\prime} \leq 0, \forall z \\
u^{\prime}=a^{\prime} t^{\prime}, v^{\prime}=0 \text { at } z^{\prime}=0 ; \\
u^{\prime}=0, v^{\prime}=0 \text { at } z^{\prime} \rightarrow \infty
\end{array}\right\} \forall t^{\prime}>0
$$

We introduce the following non-dimensional variables and parameters:

$$
\begin{aligned}
& z=\frac{w_{0}^{\prime} z^{\prime}}{v}, t=\frac{w_{0}^{\prime 2} t^{\prime}}{v}, u=\frac{u^{\prime}}{U_{0}^{\prime}}, v=\frac{v^{\prime}}{U_{0}^{\prime}}, \\
& M=\frac{\sigma B_{0}^{2} v}{\rho w_{0}^{\prime 2}}, \Omega=\frac{2 \Omega^{\prime} v}{w_{0}{ }^{2}}, a=\frac{a^{\prime} v}{w_{0}{ }^{3}}
\end{aligned}
$$

The non-dimensional form of the Equations (15) and (16) are

$$
\begin{aligned}
& \frac{\partial u}{\partial t}-\frac{\partial u}{\partial z}-\Omega v=\frac{\partial^{2} u}{\partial z^{2}}+\frac{M}{1+m^{2}}(m v-u) \\
& \frac{\partial v}{\partial t}-\frac{\partial v}{\partial z}+\Omega u=\frac{\partial^{2} v}{\partial z^{2}}-\frac{M}{1+m^{2}}(m u+v)
\end{aligned}
$$

Subject to the initial and boundary conditions:

$$
\left.\begin{array}{l}
u=0, v=0 \text { for } t \leq 0, \forall z \\
u=a t, v=0 \text { at } z=0 \\
u=0, v=0 \text { at } z \rightarrow \infty
\end{array}\right\} \forall t>0
$$

\section{Method of Solution}

Let us introduce the complex variable $q$ defined by $q=u+\mathrm{i} v$ where $\mathrm{i}^{2}=-1$.

The non-dimensional forms of the equation governing the flow can be rewritten as follows:

$$
\frac{\partial q}{\partial t}=\frac{\partial^{2} q}{\partial z^{2}}+\frac{\partial q}{\partial z}-A q
$$

where $A=\mathrm{i} \Omega+\frac{M(1+\mathrm{i} m)}{1+m^{2}}$

Subject to the boundary conditions:

$$
\left.\begin{array}{l}
q=0 \text { for } t \leq 0 \\
q=a t, v=0 \text { at } z=0 \\
q=0, v=0 \text { at } z \rightarrow \infty
\end{array}\right\} \forall t
$$

On applying Laplace Transform to the Equation (23), the following ordinary differential equation is derived

$$
\frac{\mathrm{d}^{2} \bar{q}}{\mathrm{dz} z^{2}}+\frac{\mathrm{d} \bar{q}}{\mathrm{~d} z}-(A+s) \bar{q}=0
$$

with relevant boundary conditions :

$$
\begin{gathered}
\bar{q}=\frac{a}{s^{2}} \text { at } z=0 \\
\bar{q}=0 \text { at } z \rightarrow \infty
\end{gathered}
$$

The solution of the Equation (26) under the conditions 
(27) and (28) is

$$
\bar{q}=\frac{a}{s^{2}} \mathrm{e}^{-\xi z}
$$

Taking inverse Laplace transforms of the Equation (29) we derive the following:

$$
\begin{aligned}
q & =a \mathrm{e}^{-\eta \sqrt{t}}\left[\left(\frac{t}{2}-\eta \frac{\sqrt{t}}{2 \sqrt{b}}\right) \mathrm{e}^{-2 \eta \sqrt{b t}} \operatorname{erfc}(\eta-\sqrt{b t})\right. \\
& \left.+\left(\frac{t}{2}+\eta \frac{\sqrt{t}}{2 \sqrt{b}}\right) \mathrm{e}^{2 \eta \sqrt{b t}} \operatorname{erf} c(\eta+\sqrt{b t})\right]
\end{aligned}
$$

where, $\quad b=\frac{4 A+1}{4}, \quad \xi=\frac{1+\sqrt{1+4(A+s)}}{2}, \quad \eta=\frac{z}{2 \sqrt{t}}$ and $A=\mathrm{i} \Omega+\frac{M(1+\mathrm{im})}{1+m^{2}}$

\section{Skin Friction}

The non-dimensional skin-friction at the plate is given by

$$
\begin{aligned}
& \tau=-\frac{1}{2 \sqrt{t}}\left(\frac{\partial q}{\partial \eta}\right)_{\eta=0} \\
& =\frac{a}{2}\left[\frac{1}{\sqrt{b}} \operatorname{erf}(\sqrt{b t})+2 t \sqrt{b} \operatorname{erf}(\sqrt{b t})+\frac{2 t}{\sqrt{\pi}} \mathrm{e}^{-b t}+t\right] \\
& =\tau_{x}+\mathrm{i} \tau_{y}
\end{aligned}
$$

where $\tau_{x}$ and $\tau_{y}$ are the skin-frictions at the plate due to the primary and the secondary velocity fields. The expressions for $\tau_{x}$ and $\tau_{y}$ are obtained but not presented here for the sake of brevity.

\section{Results and Discussion}

In order to get the physical insight into the problem we have carried out numerical calculations for the representative velocity field and skin-friction at the plate for different values of the physical parameters involved and these values have been demonstrated in different graphs. Our investigation is restricted to $t$ equal to 1 and the other parameters namely, rotational parameter $(\Omega)$, Hartmann number $(M)$, Hall parameter $(m)$ and accelerating parameter (a) has been considered arbitrarily.

Figure 1 depicts the variation in skin-friction $\tau_{x}$ due to the primary velocity field versus Hall parameter $m$ for different values of rotational parameters $\Omega$. It is noticed that $\tau_{x}$ decreases with increasing values of Hall parameter $m$ whereas a rise in the values rotational parameter $\Omega$ results a growth in $\tau_{x}$.

The influence of Hartmann number $M$ on skin-friction $\tau_{x}$ against Hall parameter $m$ is presented in Figure 2. It is observed that a growth in the Hartmann number $M$ leads to an increase of $\tau_{x}$. Moreover, it is seen

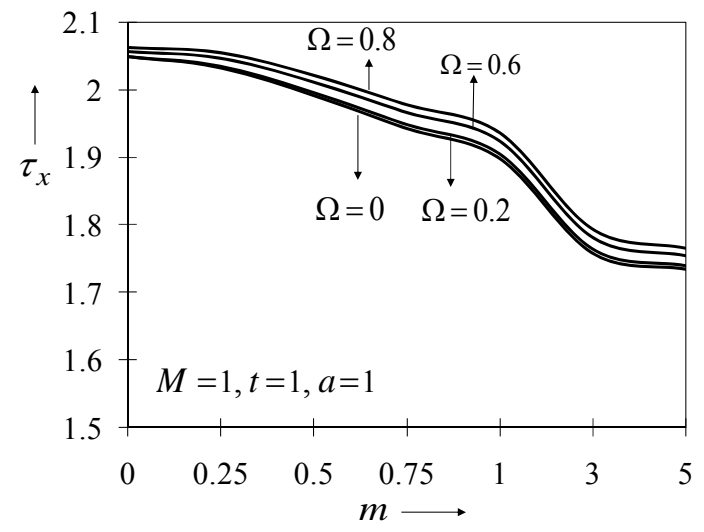

Figure 1. The behavior of $\tau_{x}$ versus $m$ under the effect of $\Omega$.

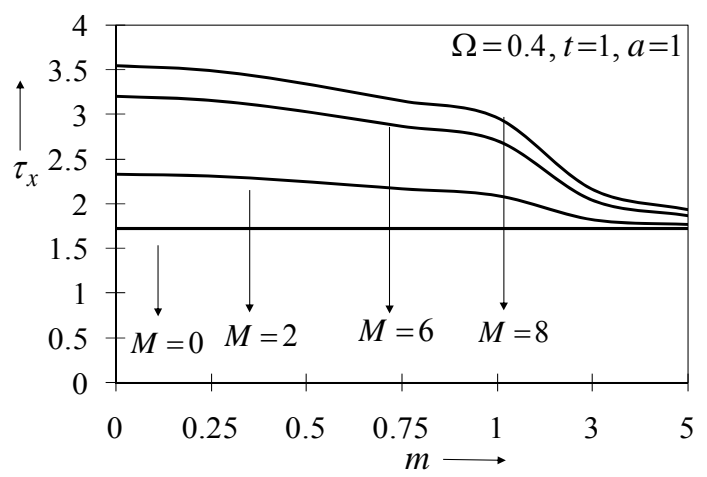

Figure 2. The behavior of $\tau_{x}$ versus $m$ under the effect of $M$.

that $\tau_{x}$ decreases very slowly and steadily as the Hall parameter $m$ rises. In other words, an increase in the Hall parameter or a decrease in Hartmann number results a decrease in drag force at the plate due to the primary velocity.

Figure 3 demonstrates the nature of $\tau_{x}$ against Hall parameter $m$ under the effect of accelerating parameter $a$. Figure 3 exhibits a substantial growth in $\tau_{x}$ with increasing values of accelerating parameter $a$. It is also seen that $\tau_{x}$ is unalterable for small values accelerating parameter or in absence of plate acceleration. That is, $\tau_{x}$ (drag force) due to the primary velocity is undisturbed whenever the plate is at rest as well for the slow movement of the plate. However, for higher values of the accelerating parameter $(a)$ skin-friction $\tau_{x}$ decreases gradually with increasing values of Hall parameter $m$.

The behaviour of $\tau_{y}$ (drag force per unit area) due to the secondary velocity field versus Hall parameter $m$ under the effects of Hartmann number $M$, rotational parameter $\Omega$ and acceleration parameter $a$ respectively are depicted in Figures 4-6. It is noticed that a rise in $M, \Omega$ and accelerating parameter $(a)$ results a growth in $\tau_{y}$. Moreover $\tau_{y}$ is undisturbed by 


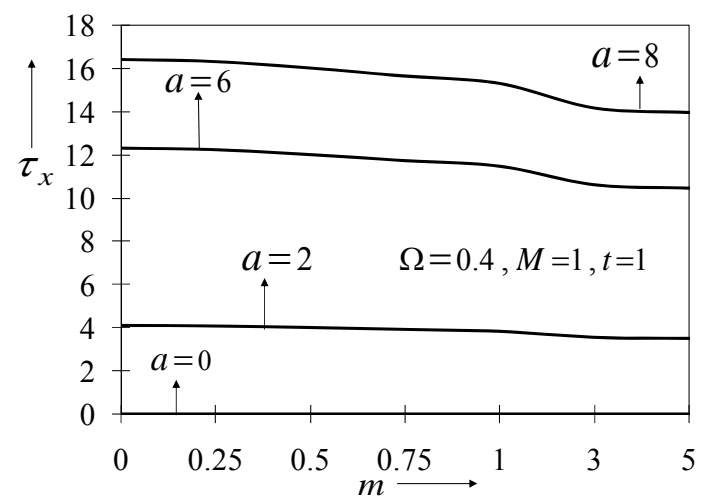

Figure 3. The behavior of $\tau_{x}$ versus $m$ under the effect of a.

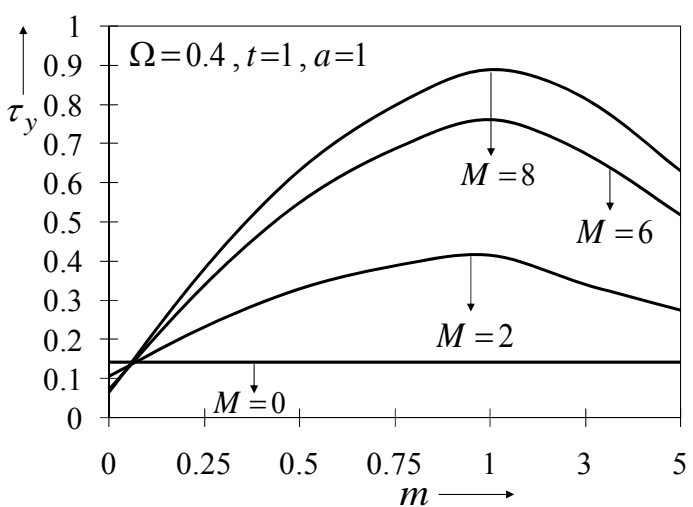

Figure 4. The behavior of $\tau_{y}$ under the effect of $M$ versus $m$.

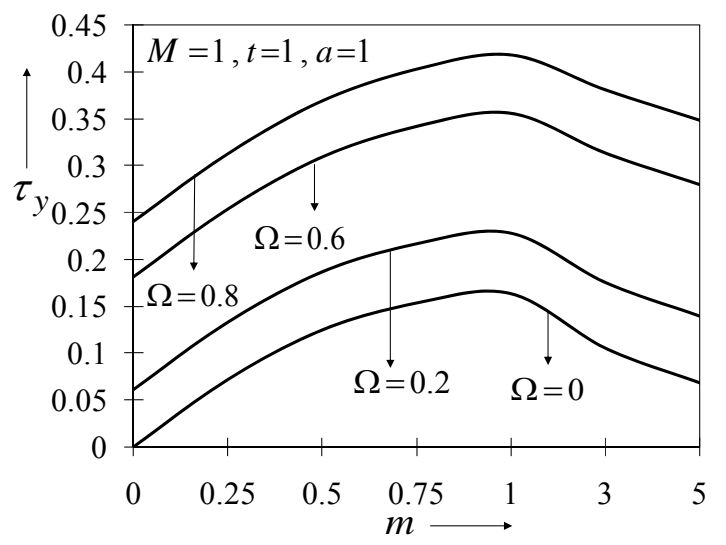

Figure 5. The behavior of $\tau_{y}$ versus $m$ under the effect of $\Omega$.

Hall effect in absence of magnetic field and acceleration of the plate. The same figures also indicate the growth of $\tau_{y}$ for small and moderate values of Hall parameter $(m \leq 1)$ and afterwards a reversal trend on $\tau_{y}$ is noticed.

The effect of Hartmann number $M$ on primary velocity field is presented in Figure 7. This figure clearly

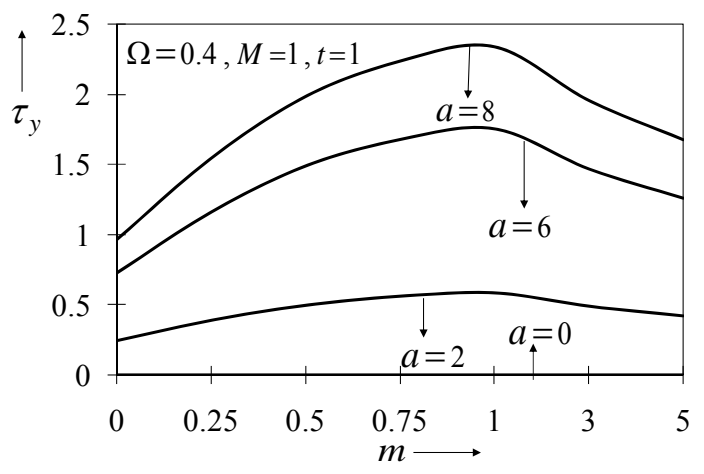

Figure 6. The behavior of $\tau_{y}$ versus $m$ under the effect of a.

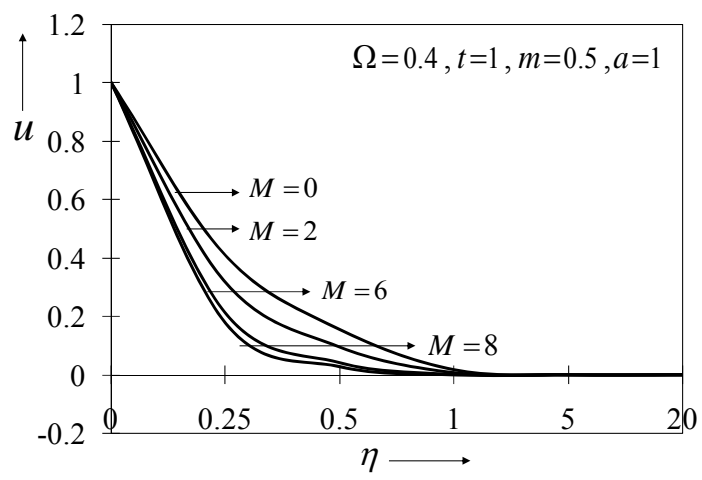

Figure 7. The variation of $u$ versus $\eta$ under the effect of M.

shows that the primary velocity sharply decreases in a thin layer of the fluid adjacent to the plate surface and then it decreases slowly and steadily to its minimum value $u=0$ as $\eta \rightarrow \infty$. The same figure also indicates that an increase in the Hartmann number $M$ has an inhibiting effect on the primary velocity $u$. The primary fluid velocity $u$ gets continuously reduced with increasing $M$. That is the application of the transverse magnetic field retards the primary motion. This phenomenon has an excellent agreement with the physical fact that the Lorentz force generated in present flow model due to interaction of the transverse magnetic field and the fluid velocity acts as a resistive force to the fluid flow which serves to decelerate the flow. As such the magnetic field is an effective regulatory mechanism for the flow regime.

Further, it is worthwhile to mention that the effect of Hartmann number $M$ on primary velocity $u$ is negligible for large $\eta$. In other words the fluid motion far away from the plate is undisturbed due to imposition of the magnetic field.

Figure 8 indicates that an increase in the plate acceleration causes the primary flow to retard comprehendsively near the plate. That is the role of accelerating parameter $a$ on primary velocity field is almost similar to 
the role of Hartmann number $M$. However, in absence of acceleration a very small growth of primary velocity is noticed in a thin fluid region adjacent to the plate surface and afterwards it falls slowly and steadily to its free stream value as $\eta \rightarrow \infty$.

Figures 9-12 demonstrate the behaviour of the secondary velocity field $(v)$ under the effects of rotational parameter $(\Omega)$, Hartmann number $(M)$, Hall parameter $(m)$ and accelerating parameter $(a)$ respectively. From these figures, it is interesting to observe that the magnitude of $v$ increases from its zero value at the plate surface into a fluid region adjacent to

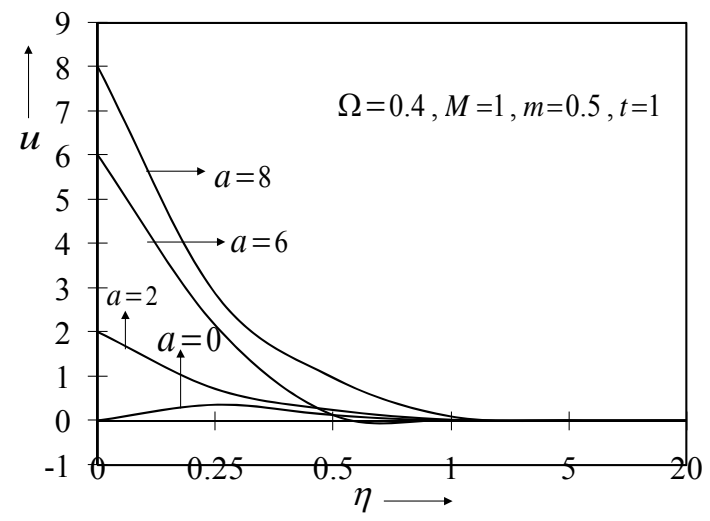

Figure 8. The variation of $u$ versus $\eta$ under the effect of a.

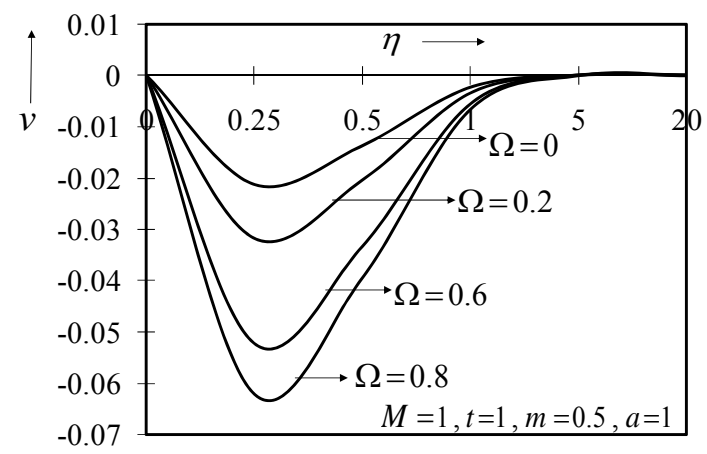

Figure 9. The behavior of $v$ under the effect of $\Omega$, versus $\eta$.

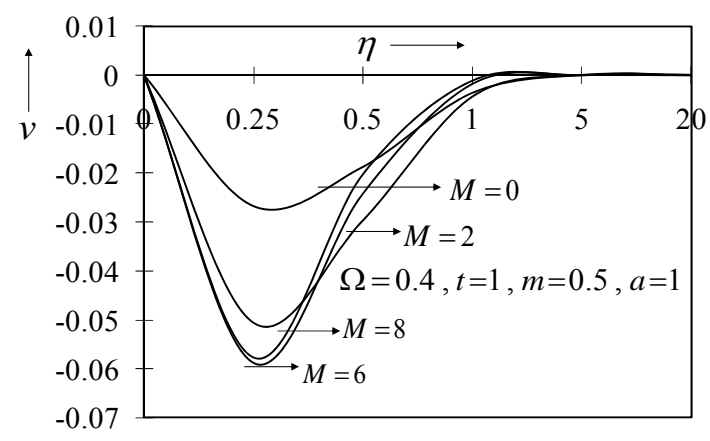

Figure 10. The behavior of $v$ under the effect of $M$, versus $\eta$.

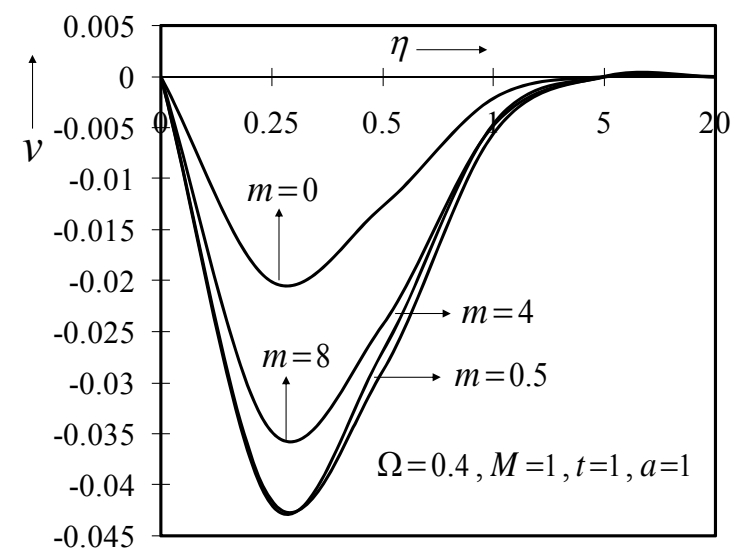

Figure 11. The behavior of $v$ under the effect of $m$, versus $\eta$.

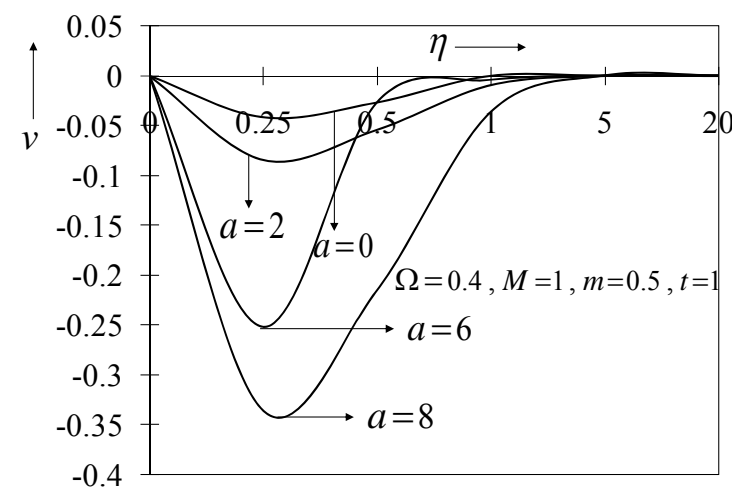

Figure 12. The behavior of $v$ under the effect of $a$, versus $\eta$.

the plate and then it decreases to zero value (attainable at possibly large distances from the plate surface). This clearly agrees with the boundary conditions of the present flow problem. Figures $\mathbf{9}$ and $\mathbf{1 2}$ indicate that a rise in the values of rotational parameter $\Omega$ and accelerating parameter $a$ causes a growth in the magnitude of secondary velocity. However, this effect seems to be negligible in the fluid region far away from the plate. Further, it is worthwhile to mention that for small and moderate values of Hartmann number $M$ and Hall parameter $m$, the behaviour of secondary velocity field is identical to its behaviour under the effects of $\Omega$ and accelerating parameter $a$ whereas, for higher values of Hartmann number $M$ and Hall parameter $m$ the behaviour of secondary velocity $v$ takes a reverse trend.

It is inferred from Tables $\mathbf{1}$ and $\mathbf{2}$ that a rise in rotational parameter $(\Omega)$, and Hall parameter $(m)$ results in a growth in the primary velocity $u$.

\section{Conclusions}

- The main flow velocity $u$ decreases with an imposition of magnetic field. 
Table 1. Behavior of $u$ under the effect of $\Omega$, versus $\eta$. $(M=1, t=1, m=0.5, a=1)$.

\begin{tabular}{cccc}
\hline$\eta$ & $u(\Omega=0)$ & $u(\Omega=0.6)$ & $u(\Omega=0.8)$ \\
\hline 0 & 1 & 1 & 1 \\
0.25 & 0.3611287 & 0.3550634 & 0.3519596 \\
0.5 & 0.1239504 & 0.1185953 & 0.1158712 \\
1 & 0.0121564 & 0.0108471 & 0.0101901 \\
5 & 0 & 0 & 0 \\
\hline
\end{tabular}

Table 2. Behavior of $u$ under the effect of $m$, versus $\eta$. $(M=1, t=1, \Omega=0.4, a=1)$.

\begin{tabular}{cccc}
\hline$\eta$ & $u(m=0)$ & $u(m=4)$ & $u(m=8)$ \\
\hline 0 & 1 & 1 & 1 \\
0.25 & 0.3508695 & 0.4021751 & 0.4063124 \\
0.5 & 0.1174215 & 0.1507741 & 0.1539457 \\
1 & 0.0110595 & 0.0168414 & 0.0175416 \\
5 & 0 & 0 & 0 \\
\hline
\end{tabular}

- An increase in accelerating parameter $a$ results in a remarkable growth in the main flow velocity $u$ in a thin layer adjacent to the plate.

- The drag force due to primary velocity $\left(\tau_{x}\right)$ rises due to rotation or imposition of the transverse magnetic field or the acceleration of the plate.

- The skin friction $\tau_{y}$ (drag force due to secondary velocity) shows a growth for increasing each of Hartmann number $M$, rotational parameter $\Omega$ as well as accelerating parameter $a$.

\section{Acknowledgements}

The Authors are grateful to the CSIR-HRDG (India) for sponsoring this work through their Research-Grant-InAid. No. 25 (0209)/12/EMR-I.

\section{REFERENCES}

[1] A. Raptis, "Mass Transfer and Free Convection through a Porous Medium by the Presence of a Rotating Fluid," International Communications in Heat and Mass Transfer, Vol. 10, No. 2, 1983, pp. 141-146. http://dx.doi.org/10.1016/0735-1933(83)90040-4

[2] A. K. Singh, "Hydromagnetic Free-Convection Flow Past an Impulsively Started Vertical Plate in a Rotating Fluid," International Communications in Heat and Mass Trans- fer, Vol. 11, No. 4, 1984, pp. 339-406.

[3] A. K. Singh, "MHD Free Convection Flow in the Stokes Problems for a Vertical Porous Plate in a Rotating System," Astrophysics and Space Science, Vol. 95, No. 2, 1983, pp. 283-289.

[4] M. Alam, M. A. Sattar and M. Mansur, "Similarity Solution of Steady MHD Free Convection and Mass Transfer Flow with Thermal Diffusion in a Rotating System," Dhaka University Journal of Science, Vol. 49, No. 2, 2001, p. 147.

[5] L. Debnath, "Exact Solutions of the Unsteady Hydrodynamic and Hydro Magnetic Boundary Layer Equations in a Rotating Fluid System," ZAMM, Vol. 55, No. 7-8, 1975, pp. 431-438.

[6] H. Alfven, "Existence of Electromagnetic-Hydrodynamic Waves," Nature, Vol. 150, No. 3805, 1942, pp. 405-406.

[7] T. G. Cowling, "Magneto Hydrodynamics," Wiley Inter Science, New York, 1957.

[8] V. C. A. Ferraro and C. Pulmpton, "An Introduction to Magneto Fluid Mechanics," Clarandon Press, Oxford, 1966.

[9] I. Pop, "The Effect of Hall Currents on Hydro Magnetic Flow near an Accelerated Plate," Journal of Mathematical and Physical Sciences, Vol. 5, 1971, pp. 375-379.

[10] M. Kinyanjui, J. K. Kwanza and S. M. Uppal, "Magneto Hydrodynamic Free Convection Heat and Mass Transfer of a Heat Generating Fluid Past an Impulsively, Started Infinite Vertical Porous Plate with Hall Current and Radiation Absorption," Energy Conversion and Management, Vol. 42, No. 8, 2001, pp. 917-931. http://dx.doi.org/10.1016/S0196-8904(00)00115-1

[11] M. Acharya, G. C. Das and L. P. Singh, "Hall Effect with Simultaneous Thermal and Mass Diffusion on Unsteady Hydro Magnetic Flow near an Accelerated Vertical Plate," Indian Journal of Physics B, Vol. 75, No. 1, 2001, pp. 68-70.

[12] N. Ahmed and D. Kalita, "Transient MHD Free Convection from an Infinite Vertical Porous Plate in a Rotating Fluid with Mass Transfer and Hall Current," Journal of Energy, Heat and Mass Transfer, Vol. 33, No. 1, 2011, pp. 271-292.

[13] N. Ahmed, H. Kalita and D. P. Barua, "Unsteady MHD Free Convective Flow Past a Vertical Porous Plate Immersed in a Porous Medium with Hall Current, Thermal Diffusion and Heat Transfer," International Journal of Engineering, Science and Technology, Vol. 2, No. 6, 2010, pp. 59-74.

[14] N. Ahmed and H. K. Sarmah, "MHD Transient Flow Past an Impulsively Started Horizontal Porous Plate in a Rotating System with Hall Current," International Journal of Applied Mathematics and Mechanics, Vol. 7, No. 2, 2011, pp. 1-15. 
Units for Physical Properties

\begin{tabular}{|c|c|c|}
\hline Symbol & Quantity & SI unit \\
\hline$a^{\prime}$ & Acceleration of the plate & $\mathrm{m} / \mathrm{s}^{2}$ \\
\hline$a$ & Dimensionless acceleration & \\
\hline$B_{0}$ & magnetic flux density, magnetic induction & Tesla \\
\hline$m$ & Hall parameter & \\
\hline$M$ & Hartmann number & \\
\hline$t^{\prime}$ & time & $\mathrm{s}$ \\
\hline$t$ & Dimensionless time & \\
\hline$(\bar{u}, \bar{v}, \bar{w})$ & Velocity components & $\mathrm{m} / \mathrm{s}$ \\
\hline$(u, v, w)$ & Dimensionless velocity components & \\
\hline$w_{0}$ & Suction velocity & $\mathrm{m} / \mathrm{s}$ \\
\hline$(\bar{x}, \bar{y}, \bar{z})$ & Cartesian coordinates & $\mathrm{m}$ \\
\hline$\Omega^{\prime}$ & Angular velocity & $\mathrm{s}^{-1}$ \\
\hline$\Omega$ & Dimensionless angular velocity & \\
\hline$\rho$ & Fluid density & $\mathrm{kg} / \mathrm{m}^{3}$ \\
\hline$\sigma$ & Electrical conductivity & $(\text { ohm } \times \text { meter })^{-1}$ \\
\hline$v$ & Kinematic viscosity & $\mathrm{m}^{2} / \mathrm{s}$ \\
\hline$\left(\tau_{x}, \tau_{y}\right)$ & Components of skin friction & \\
\hline
\end{tabular}

